\title{
Femtosecond Dynamics - Snapshots of the Early Ion-Track Evolution
}

\author{
G. Schiwietz, K. Czerski, M. Roth, \\ F. Staufenbiel, and P.L. Grande\# \\ Hahn-Meitner-Institut, Abteilung SF4, \\ Glienicker Str. 100, 14109 Berlin, Germany \\ \# Instituto de Física, Universidade Federal do \\ Rio Grande do Sul, 91500 Porto Alegre, Brazil \\ corresponding author: G. Schiwietz \\ (ph.: +49-30-8062-2448, e-mail: schiwietz@hmi.de) \\ PACS:79.20.Rf,31.70.Hq,79.20.Fv,72.20.Jv,32.80.Hd,72.15.Lh \\ keywords: electron temperature, ion-track potential, \\ multiple ionization, neutralization, recombination, \\ Auger decay, electron-spectra
}

November 27th 2003

\begin{abstract}
The energy dissipation and femtosecond dynamics due to fast heavy ions in matter is critically reviewed with emphasis on possible mechanisms that lead to materials modifications. Starting from a discussion of the initial electronic energy-deposition processes, three basic mechanisms for the conversion of electronic into atomic energy are investigated by means of Auger-electron spectroscopy. Results for amorphous Si, amorphous $\mathrm{C}$ and polypropylene are presented and discussed. Experimental evidence for a highly charged track region as well as for hot electrons inside tracks is shown. As follows mainly from Auger-electron spectroscopy, there are strong indications for different track-production mechanisms in different materials.
\end{abstract}




\section{Introduction}

A fast heavy ion may lead to permanent material changes in a small volume surrounding the virtually straight ion path. The high electronic energy deposition gives rise to the formation of a chemical or structural defect cluster of cylindrical shape with an extremely large aspect ratio exceeding 1:1000. Such a defect cluster and its electronic and atomic precursors are denoted ion track. The appearance of track effects in polymers is known since some decades [1] and has found widespread applications in the meanwhile [2]. Nowadays it is known that other insulators and even metallic glasses [3] are also subject to materials modifications by heavy ions. There are, however, a few seemingly contradictory models for the track-production mechanisms and until now most of them cannot completely be ruled out. In order to reveal the present status and the weak points of our present knowledge, a review of the possible scenarios of the track evolution is given. Special emphasis is devoted to the short-time phenomena from the initial excitation and ion-energy loss processes to the electronic deexcitation processes.

Fig. 1 displays a schematic view of the time dependence of the ion track evolution. The upper part shows the rapidly passing projectile (dashed arrow). Once the projectile has reached its equilibrium charge state, there will be only minor fluctuations of its internal state and it will move with constant velocity along a straight-line trajectory until deep inside the solid. Thus, the projectile ion acts as a well defined and virtually instantaneous source of strongly localized electronic excitation. About $50 \%$ of the total electronic energy is deposited inside the so-called infra-track radius of about $1 \mathrm{~nm}$ around the projectile path at projectile energies of a few $\mathrm{MeV}$ per nucleon. Excitation times are $10^{-19}$ to $10^{-17} \mathrm{~s}$ for inner-shell processes and reach $10^{-16} \mathrm{~s}$ for plasmon production.
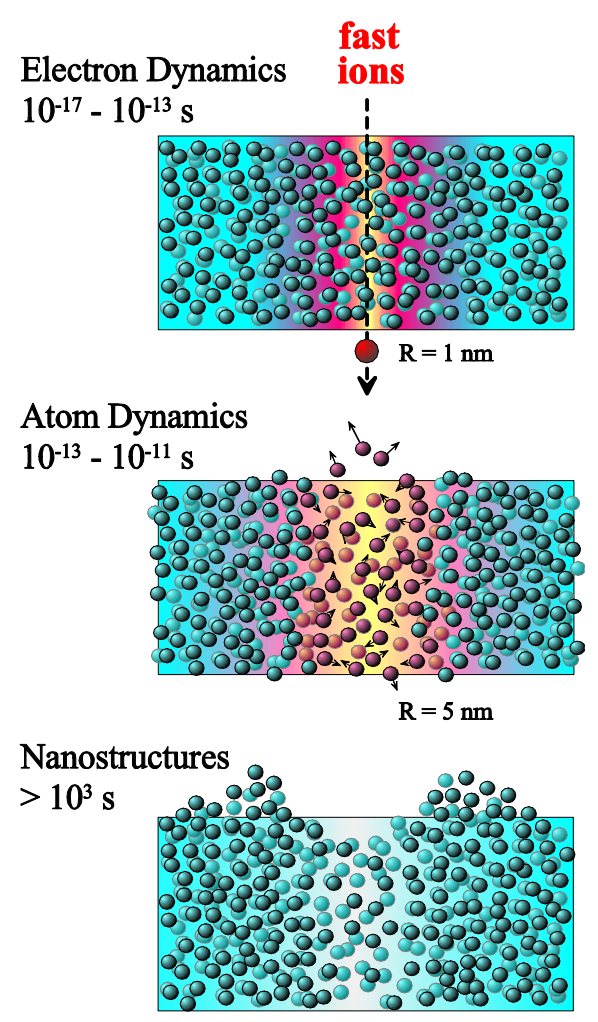

Figure 1: Time evolution of an ion track. The initial excitation and ionization of atoms induces atomic motions, which freeze out and may lead to permanent rearrangements. In the bulk this may lead to structural or chemical modifications. At the surface craters or blisters on an atomic scale can be produced.

After these initial ionization and excitation events, the electronic system evolves further. The most important parameters that drive subsequent the solid-state evolution are the local electron-energy density and the local ionization density, as will be explained below. Most experimental techniques do not have direct access to these quantities. The closely related total ion- 
energy loss and the degree of innershell ionization, however, are subject of many investigations and they are discussed in sections 2 and 4 for the case of fast heavy ions.

After the initial energy-transfer by heavy ions, electrons have escaped and the center of the track is highly ionized. Depending on the ionization density and on the charge-neutralization time the mutual repulsion of positively charged target ions may convert a significant amount of the stored electronic potential energy into atomic motion. This conversion mechanism is described by the Coulomb-explosion model $[1,4,5,6]$ and the corresponding electrostatic potential is discussed in section 5. Coulomb explosion will be significant only if the chargeneutralization time exceeds $10^{-14} \mathrm{~s}$ for light target atoms and $10^{-13} \mathrm{~s}$ for heavy atoms.

Perturbation theory predicts neutralization times of about $10^{-16} \mathrm{~s}$ (given by the inverse plasmon frequency) for a weak and homogeneous charge displacement in free-electron gas-like metals, such as Al. Thus, for most metals charge neutralization might be fast and Coulomb explosion is impossible. Nevertheless, one has to consider that there is an extremely high charge density at the center of heavy-ion tracks. Furthermore, the spatial density of excited plasmons might already be saturated due to the passage of the ion. For highly charged ions such effects go beyond perturbation theory and might have a severe influence on the collective electron properties. Thus, an experimental determination of charge-neutralization times is needed in order to judge about the importance of the Coulomb-explosion mechanism.

Even if charge-neutralization is rapid, electronic recombination might still be slow, leading to a hot electron gas at the center of the track. Two different mechanisms may then convert this internal electronic energy (quantified by an electron temperature, as discussed in section 6) into atomic motion.

- The lattice-relaxation model $[7,8]$ describes a collective atomic rearrangement due to (predominantly repulsive or antibinding) non-equilibrium interatomic potentials. Thus, a fraction of the electronic potential energy, or equivalently the degree of target excitation, leads to modified inter-atomic forces and subsequent atomic motion in this model.

- The electronic thermal-spike model $[9,10,11,12,13,14]$ assumes that electronic excitation leads to the formation of a hot plasma and, via the electronphonon coupling (equivalent to electron-atom collisions), to an increased thermal atomic motion. Thus, except for the efficiency of the electron-phonon coupling, the mean kinetic electron energy is the main ingredient in this model.

The relative importance of the three mechanisms depends on the charge-neutralization time, on the strength of the modified inter-atomic forces and on the electron-phonon coupling constant. For highly excited ion tracks, all of these quantities are uncertain or even unknown and thus, the influence of a certain mechanism can only be determined experimentally. This, however, is complicated by the fact that atomic motion in solid matter may be converted into a stochastic motion on a time scale of $10^{-13}$ to $10^{-12} \mathrm{~s}$, largely independent of the early stage of the evolution. This situation is depicted in the center part of Fig. 1. Furthermore, slow atomic 
relaxation processes, such as recrystallization, and the influence of local structures and delay times on phase transitions may prohibit any definite conclusions. Hence, there seems to be no way to distinguish between the models on a pure experimental basis, if only the resulting modified material properties such as in the lower part of Fig. 1 are investigated.

One possible way to improve the interpretation of material modification effects is the investigation of prompt emitted 'particles' that carry information from inside the track. Ejected electrons or x-rays can be used as precursors of the corresponding transient material states. Electrons may be probes for the first $10^{-18}$ to $10^{-14} \mathrm{~s}$ of the track formation and energy dissipation. For reviews on transport of fast electrons and fast-ion-induced electron emission from solids, the reader is referred to refs. [15, 16, 17, 18].

\section{Initial Interaction Processes, Charge States and Ion Energy-Loss}

In this section, the initial interaction processes and projectile-related quantities will be discussed. Regarding the state of the projectile, inelastic collision processes depend on

- the projectile nuclear charge $\mathrm{Z}_{p}$.

- the projectile charge-state $\mathrm{q}_{p}$ and only to a minor extend on the degree of internal projectile excitation inside the solid.

- the dynamic projectile screening (dressed projectile potential) due to the polarization of target valence- or conduction-band electrons as well as inner-shell electrons.
- the projectile velocity $\mathrm{v}_{p}$. Throughout this paper, $\mathrm{v}_{p}$ will be given in units of the Bohr velocity $\mathrm{v}_{B}$ of $2.19 \times 10^{6} \mathrm{~m} / \mathrm{s}$ corresponding to $25 \mathrm{keV} / \mathrm{u}$ or equivalently 1 atomic unit (a.u.).

In solids there is no open experimental access to most dynamic quantities regarding the projectile as well as the target. Hence, it seems appropriate to discuss the corresponding processes for individual ion-atom collisions, where the cross sections for all singleelectron transitions are reasonably well known and understood. Before we look closer at these cross sections, however, we should first consider the differences between thin gas targets at moderate pressures and solid-state targets at typical densities. At room temperature for a gas pressure of $1 \mathrm{~atm}$ the atomic gas density is by three orders of magnitude lower than the corresponding solid-state density. Exactly this difference in the mean inter-atomic distances is solely responsible for all solid/gas differences. It leads to the following effects:

1. Inside solids the collision frequency is enhanced by 6 orders of magnitude in comparison to a gas target at typical gas-cell pressures. Beyond for the pure statistical enhancement of transition rates, this leads to the ionization of excited states that are populated in a previous collision. Thus, electrons in excited states are stripped off before they may decay to the ground state inside solids. In gas targets such highly excited projectile states decay preferentially via an x-ray transition before the next collision takes place, thereby stabilizing the lower projectile charge state. Especially for fast heavy ions this difference results in significantly increased projectile charges and 
stopping forces in solids $[19,20$, $21,22]$.

2. Inside solids the level structure is modified due to the presence of neighboring atoms. For inner shells the relative effect is small, but for valence bands there is a considerable influence of electron hopping and the energy gaps may vanish. The influence of the gap on the energy loss is important only at low velocities, where mainly valence electrons are involved [23].

3. Inside solids collective excitations (plasmons) appear as a new energy-loss mechanism. For fast projectiles, however, this excitation mode suppresses the dipoletype atomic excitation processes via the plasmon screening (wake potential) discussed below. Thus, for fast ions the total energy loss as well as its impact-parameter dependence are barely influenced by the plasmon screening.

4. Inside solids there is a dynamic projectile screening due to electrons of the valence and conduction bands that are attracted by the positively charged projectile. This collective effect reduces the strength of the projectile/electron interaction. At low velocities it is described by the Thomas-Fermi screening-length and at high velocities it results in the so-called wake potential related to plasmon excitation [24]. As mentioned above, there is a cancellation of effects for swift particles. Thus, the screening effect becomes important only at low velocities [25], where plasmon excitation is suppressed.

Summarizing the above remarks, two marked differences between the energy losses in solids and individual atoms may be noted. Slow light ions involve reduced energy losses due to the longranged solid-state screening (see item 4 above). More important for the subject of this review, however, is the behavior of swift heavy ions. For these ions, the energy loss in solid is enhanced due to increased charge states (see item 1 above). Thus, atomic cross sections should yield a reliable picture of the direct ion-solid interaction processes for fast heavy ions as long as we consider the differences in the chargestate distributions.

Fig. 2 displays schematic cross sections for all basic single-electron reactions in ion atom collisions. Actual numbers for these total cross sections have been taken from different sources for protons on atomic hydrogen and also on helium. The scaled velocity $\mathrm{v}_{p} / \mathrm{v}_{o}$ corresponds to a certain selected target-electron shell with the mean orbital velocity $\mathrm{v}_{o}$. For conduction-band electrons $\mathrm{v}_{o}$ may be replaced by $2 / 3 v_{F}$, where $\mathrm{v}_{F}$ is the Fermi velocity. The cross section values are given in arbitrary units, since there is no simple scaling that covers the full range of small $\left(\mathrm{v}_{p} / \mathrm{v}_{o} \ll 1\right)$ as well as large $\left(v_{p} / v_{o} \gg 1\right)$ velocities. Note that the displayed dependencies are typical for a fixed projectile charge-state. The partial stopping cross sections have shown in the upper part of the plot are derived from the total cross section by multiplication with the average energy related to the different processes.

One may see that the excitation and ionization cross sections in Fig. 2 behave quite similar. They are rising from low energies towards higher ones until a maximum around $\mathrm{v}_{p}=\mathrm{v}_{o}$ is reached. At higher velocities these cross sections drop with velocity and asymptotically they are proportional to $Z_{p}^{2} \ln \left(v_{p}^{2}\right) / v_{p}^{2}$. The cross-section reduction at high velocities is mainly due to the reduced interaction time. In 


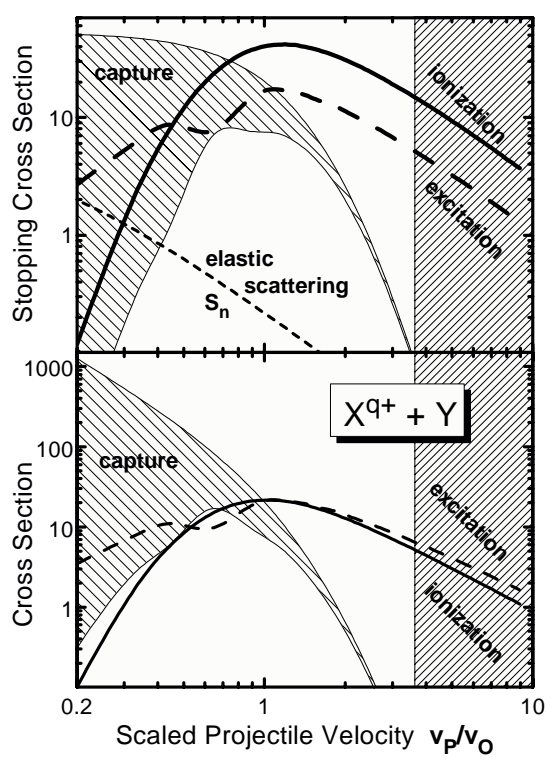

Figure 2: Typical behavior of basic single-electron processes as a function of the reduced projectile velocity $v_{p} / v_{o}$. The velocity-scaling parameter $v_{o}$ is the mean orbital velocity for a specific inner-shell or valence-band electron. The energy dependence for excitation, ionization and electron capture cross-sections are shown in the lower plot. The corresponding stopping cross-sections are shown in the upper plot, together with the so-called nuclear energy loss due quasi-elastic projectile scattering.

other words, the probability for hitting a target electron is significantly reduced at high projectile energies. At intermediate energies ions slow down due to a manyfold of different processes, all of similar importance. There are high transition probabilities at small impact parameters and even multi-electron transitions may dominate the collision process [26].

At low velocities the excitation and ionization cross sections drop, since the target electron may adjust adiabati- cally to the projectile motion. A transient quasi-molecular orbital is formed and when both collision partners separate there is a high probability for the electron to return back to its ground state. For specific orbitals, however, quasi-molecular promotion and rotational coupling may still lead to exceptional high transition probabilities. In this energy range the collision dynamics depends significantly on the projectile charge state, impact parameter and on the specific projectile-target combination [27].

The capture cross section is indicated by the hatched area on the lefthand side in Fig. 2. It falls drastically with increasing energy, since electron capture requires that a slow target electron adjusts to the high projectile speed (jumping onto a moving train). This is possible only at small impact parameters, where the electron velocity may exceed the mean orbital velocity. Asymptotically the electrontransfer cross section is proportional to $q_{p}^{5} / v_{p}^{11}$ as predicted by second-order perturbation theory.

At low ion speeds electron capture is very sensitive to the details of the level structure of both collision partners. The upper and lower boundaries of the hatched area in the graph are representative for protons interacting with atomic hydrogen, respectively with helium atoms. In the first case $\left(\mathrm{H}^{+}+\mathrm{H}\right)$ an electron may be transferred in a resonant process, keeping the binding energy unchanged. Thus, tunneling slightly below the potential barrier between projectile and target is the dominant reaction process. The second case $\left(\mathrm{H}^{+}+\mathrm{He}\right)$ requires an energy transfer that is very unlikely, especially at large impact parameters. This leads to orders-of-magnitude reductions for the non-resonant electroncapture probabilities. At low projectile energies target as well as projectile excitation and ionization are also sig- 
nificantly dependent on the details of the collision system and especially for outer-shell processes no simple scaling rules apply. The corresponding crosssection variations are typically less pronounced than for the capture process. For the sake of simplicity these variations are not shown in the figure.

So far we have considered only an individual target-electron shell in the above discussion. Let us now turn to the more global behavior. At high energies, where the projectile is much faster than even the target K-shell electrons, all electrons contribute very similar to the projectile energy loss. The excitation and ionization cross sections are significantly reduced for deeply bound target shells, but the mean energy transfers are enhanced in an approximately reciprocal manner. Thus, the binding energy is of minor importance for electrons below a certain threshold binding-energy. At high energies the effect due to all shells may be summarized by a shell-averaged mean target-excitation energy as it appears in the Bethe logarithm of Bethe's famous stopping-power formula [28, 29]. Using such an averaged energy, the corresponding averaged orbital velocity would scale with the square root of the target nuclear charge $\mathrm{Z}_{t}$. At intermediate projectile velocities, however, inner shells do not contribute to the energy loss. Thus, a much weaker $\mathrm{Z}_{t}$ dependence of the averaged velocity is expected close to the stopping power maximum. One may estimate that the stopping power maximum in Fig. 2 varies only between about 50 and $300 \mathrm{keV} / \mathrm{u}$ for solid-state targets of the whole periodic table, but restricted to light ions. For proton beams this estimated energy range matches the experimental results to within a factor of two.

As indicated in the discussion further above, however, the variation of the projectile charge-state is very im- portant and has to be considered for projectiles with higher nuclear charges. The hatched square on the right-hand side of the figure corresponds roughly to the experimental stopping-power maxima for heavy ions if we consider the shell-averaged velocity scaling parameter for the x-axis. This seems to be in contradiction with the plotted partial stopping-power curves (with their maximum close to $\mathrm{v}_{p}=\mathrm{v}_{o}$ ), but one has to keep in mind that the projectile charge-state variation with velocity is not included in these curves. Nevertheless, for the velocity regime where ion tracks are produced (hatched square) one may summarize as follows. Electron capture and projectile-electron loss are important only insofar as they determine the projectile charge state. Target ionization processes, often with high energy transfers, clearly dominate the shellaveraged stopping force.

For few-electron systems $(\mathrm{H}+\mathrm{H}$ or $\mathrm{H}+\mathrm{He})$, there exist accurate quantum-mechanical solutions of the time-dependent Schrödinger equation, yielding stopping powers that agree to within a few percent with the experimental data. The remaining discrepancies may even be traced back to result from the neglect of electron-correlation effects [30, 31]. Also impact-parameter dependent ionization probabilities and electronic energy transfers are in reasonable agreement with available experimental data [32]. The situation is less satisfying for many-electron systems as they are typical for ion-solid interactions. However, the whole treatment simplifies again if we restrict ourselves to fast projectiles. As shown above, fast ions loose their kinetic energy mainly through ionization of atoms from all shells and to some extent through excitation of valence-and conduction-band states. Although the current experimental uncertainties of 
energy-loss determinations are much below the theoretical uncertainties, these energy-loss mechanisms are expected to be qualitatively well understood.

Different attempts for a precise description of the energy loss of swift ions in multi-electron targets are currently being worked out in the frame work of simplified models $[33,34,35,36,37]$. They rely on an explicit consideration of the different projectile charge states and the corresponding projectile screening due to bound electrons. Furthermore, they include projectile excitation and ionization processes as well as higher order terms that go beyond perturbation theory. Especially for heavy ions, accurate equilibrium charge-states are needed if the energyloss prediction should reach a precision below $10 \%$. For heavy ions at $5 \mathrm{MeV} / \mathrm{u}$ such charge-state data are presented and discussed in the next paragraphs.

Fig. 3 displays experimental as well as theoretical mean projectile chargestates $\bar{q}$ for different carbon foils of thicknesses between 4 and $50 \mu \mathrm{g} / \mathrm{cm}^{2}$. The experiments have been performed with a stripper foil in a focal point a few $m$ downstream of the ISL heavyion cyclotron [38]. The charge-state distributions have been measured by using a dipole magnet coupled with a quadrupole triplet to focus each selected final charge-state fraction of the beam into a widely open Faraday cup. The resulting uncertainties are typically below 0.2 charge units for a stable beam and a homogeneous stripper foil. The initial charge states are indicated as arrows on the left axis.

The projectile charge-state is determined by the balance of electron capture and projectile-electron loss as well as by the excitation, Auger and optical decay of excited projectile states. A few single-electron models exist for the solution of the rate equations that govern the evolution of the projectile-state

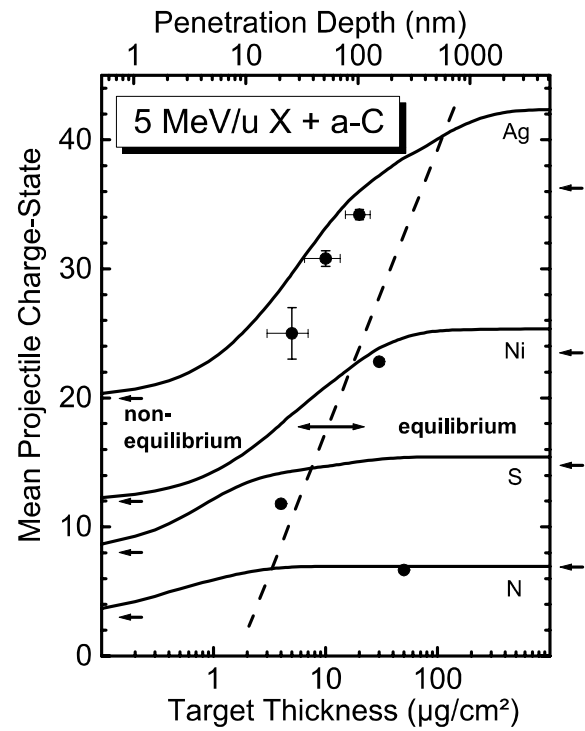

Figure 3: Measured mean projectile charge-states $\bar{q}$ for different ion species as a function of the target thickness in comparison with theoretical predictions [39]. The arrows on the left-hand side indicate the initial charge states. The arrows on the right-hand side of the frame indicate the mean equilibrium charge-states as determined from an accurate fit to a nearly complete set of published data.

population. Only one code, however, is known to us that allows to treat projectiles with up to about 9 bound electrons [39]. Explicit consideration of all sublevels of the K, L, and M shells and all possible populations for all charge states would require to solve an unfeasible amount of coupled rate equations with the corresponding transition rates. Using reasonable assumptions, this system of equations is reduced to only 81 coupled equations, which are solved numerically. Results of this model are depicted as solid curves in Fig. 3. 
Fitted equilibrium charge-states $\bar{q}$ for the limit of large target thicknesses are indicated as arrows on the right side of plot. These precision chargestates fits are discussed further below.

The solid curves show a monotonously increasing mean charge state with increasing target thickness. These curves overestimate the experimental data and the equilibrium-fit results by up to 6 charge units for the heaviest projectile ion. These deviations are most likely due to uncertainties of the calculated transition cross-sections. They point to underestimated capture cross sections or overestimated electron-loss cross sections. The theoretical curves as well as the experimental data for $\mathrm{Ag}$ show the importance of non-equilibrium charge-states, which are directly related to non-equilibrium energy losses in thin-film experiments. For heavy ions at a few $\mathrm{MeV} / \mathrm{u}$ stopping powers might be strongly reduced (roughly proportional $q^{2}$ ) in the first few hundred nm. An approximate boarder line between non-equilibrium and equilibrium thickness at $5 \mathrm{MeV} / \mathrm{u}$ is indicated by the broken line in Fig. 3. Before we turn to a specific property of heavy ion stopping at equilibrium, however, we should discuss equilibrium charge states in somewhat more detail.

Qualitatively the mean projectile charge state is given by the Bohr stripping criteria which states that all projectile electrons with orbital velocities below the projectile velocity are stripped off. This means at equilibrium we have $v_{p} / v_{o} \lesssim 1$ for the outermost bound projectile electron. In fact, for protons in hydrogen there is a crossing of the cross sections for resonant capture and ionization (similar to the crossing in the lower graph of Fig. 2) at $v_{p} / v_{o} \approx 1.4$. Thus, the mean charge state is 0.5 at this velocity, since the electron capture and loss rates are about equal.

For highly charged heavy projectiles the electron-loss cross sections decrease with the outer-shell bindingenergy and hence, with the projectile charge. The maximum capture cross-sections on the other hand increase significantly with the projectile charge, but compared to Fig. 2 there is a steeper velocity dependence at high energies. This charge-state dependent behavior of the capture cross section stabilizes the critical velocity ratio $v_{p} / v_{o}$ where capture and loss involve equal cross sections. Typical critical ratios are $0.9<v_{p} / v_{o}<1.7$

From this discussion it is obvious that the Bohr stripping criteria should not be taken too serious. Furthermore, as discussed above, it would require enormous theoretical efforts to handle projectiles carrying many electrons in an ab-initio treatment. Therefore accurate charge-state predictions for fast heavy projectiles do still rely on semi-empirical fits to experimental data. The results of an advanced charge-state fit are described in the following.

Fig. 4 displays experimental data for the reduced projectile charge $\bar{q} / Z_{p}$ as a function of a general velocity scaling-parameter $x$. Bohr has proposed a velocity scaling-parameter $x=$ $Z_{p}^{-2 / 3} v_{p} / v_{B}[40]$. We have checked that the use of this scaling leads to average uncertainties of 1.7 charge units $( \pm 5.1 \%)$ in comparison to the available experimental data. Stopping powers derived from the Bohr scaling would be uncertain by $\pm 10 \%$, even if an otherwise perfect energy-loss theory is used. Thus, we have decided to search for a more accurate scaling of the mean charge states. A multi-parameter least-square fit [21] has been applied to published solid-state data for about 840 experimental data points. Protons and helium ions above a veloc- 


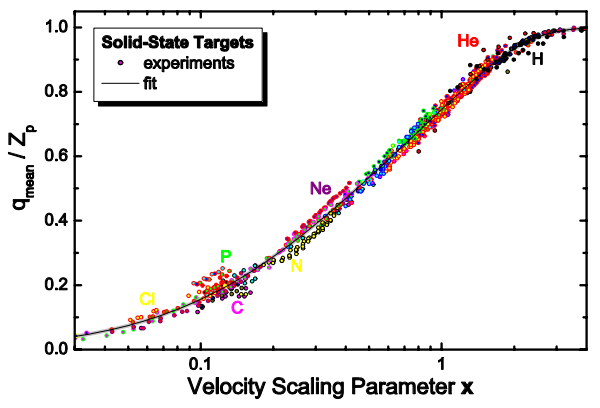

Figure 4: Measured mean equilibrium projectile charge-states $\bar{q}$ divided by the corresponding projectile nuclear charges $\mathrm{Z}_{p}$ for all ion species and all solid-state targets as a function of the scaling variable $x$ (see text). $\mathrm{Z}_{p}$ is color-coded allowing to separate the different data sets. The solid curve is an accurate fit to this nearly complete set of published charge-state data.

ity of $v_{p} / v_{B}=2$ and all heavier ions above $v_{p} / v_{B}=0.4$ have been considered. For slower projectiles we find significant deviations from simple scaling properties and band-structure effects seem to be of importance. Here we present charge-state formulas with asymptotic dependencies that are improved with respect to previous results [21]. Furthermore, resonance effects and in addition also shell-structure effects have been considered in an iterative fitting procedure, resulting in

$$
\bar{q}=\frac{Z_{p}\left(8.29 x+x^{4}\right)}{0.06 / x+4+7.4 x+x^{4}}
$$

with the scaling variable $x$

$$
x=c_{1}\left(\tilde{v} / c_{2} / 1.54\right)^{1+1.83 / Z_{p}},
$$

the two correction terms

$$
\begin{gathered}
c_{1}=1-0.26 e^{-Z_{t} / 11} e^{-\frac{\left(Z_{t}-Z_{p}\right)^{2}}{9},}, \\
c_{2}=1+0.030 \tilde{v} \ln \left(Z_{t}\right),
\end{gathered}
$$

and with the scaled projectile velocity

$$
\tilde{v}=Z_{p}^{-0.543} v_{p} / v_{B} .
$$

The 4 numerical parameters in Eq. 1 where determined at each step of the optimization by an automatically weighted least-square fit that minimizes the absolute charge-state deviation. The remaining 7 parameters in Eqs. 2 to 5 are to a large extend independent of each other and where varied manually. The power term in Eq. 2 serves to adjust the steepness of the charge-state curves as a function of $x$. It modifies the scaling behavior at small projectile nuclear charges. The correction term $c_{1}$ accounts for resonant electron capture which reduces the mean charge state $\bar{q}$ or equivalently $x$ for symmetrical projectiletarget combinations. The correction $c_{2}$ accounts for a target dependent deformation of the charge-state curves at high velocities.

The main deviation of our fit result from the Bohr scaling is the exponent -0.543 in Eq. 5. Due to the $\mathrm{Z}_{p}$ dependence in Eq. 2 the exponent is effectively reduced to about -0.46 in the vicinity of $x=0.5$. This exponent is very close to -0.45 found by Nikolaev and Dmitriev [41] for heavy ions, but far from $-2 / 3$ predicted by Bohr [40].

A mean squared deviation of about 0.37 charge units is reached with formulas 1-5. With consideration of shellstructure effects, similar as shown in ref.[21], the mean squared deviation from the experimental data is reduced to 0.28 charge units. Note that our ab-initio stopping power code CASP is now based on the above formulas and yields also charge states for arbitrary projectile/target combinations including target dependent shell effects [33, 34]. Already our previous charge-state results [21] (less certain by roughly a factor of two) have been shown to yield accurate stopping powers for $\mathrm{MeV} / \mathrm{u}$ ions in carbon [37]. An analysis of 29 overlapping data points measured in different experiments with carbon targets shows that the pure ex- 
perimental uncertainty is already 0.21 charge units. Considering this experimental error, we expect the absolute accuracy of the current fit to be about 0.2 charge units. Thus, it is hardly possible to improve the above fit without applying experimental reliability factors. Stopping powers derived from the current scaling include an error of only about $\pm 2 \%$ due to the charge-state uncertainty.

Let us now discuss electronic stopping forces in the region of high electronic energy depositions, as they are most important for track production. Here we will concentrate only on the question at which projectile energy one may expect the stopping power maximum for a specific type of ion. This energy is, e.g., important if one tries to distinguish between materials modification processes due to either electronic $\left(\mathrm{S}_{e}\right)$ or the quasi-elastic nuclear $\left(\mathrm{S}_{n}\right)$ energy losses. Often measurements are performed for projectile energies corresponding to a fixed electronic energy loss on both sides of the stopping-power maximum.

Fig. 5 displays energy-loss crosssections for $\mathrm{He}, \mathrm{Si}$ and $\mathrm{Au}$ ions in $\mathrm{C}$, Si and $\mathrm{Au}$ targets under equilibrium conditions. These projectile/target combinations cover a large fraction of the periodic table. The energy loss values stem from fits to experimental data and are obtained using the SRIM2003 code [42]. The ordinate in Fig. 5 is scaled just for convenience, in order to reduce the order-of-magnitude variations of the stopping power values.

A particularly simple scaling was used for the $\mathrm{x}$-axis in Fig. 5. Here the kinetic energy per nucleon is divided by the projectile nuclear charge $Z_{p}$. It is seen that the stopping power maxima of the different collision systems coincide approximately. The mean energy positions for the chosen projectile/target combinations correspond to about $E_{p} / M_{p}=Z_{p} \cdot 80 \mathrm{keV} / u$ and the variation around this value is about $\pm 50 \%$. Although this relation looks very simple, the explanation of this behavior is not trivial as will be discussed in the following.

Let us first concentrate on the target dependence of the stopping-power maximum for incident protons. Typical atomic outer-shell binding energies of metal atoms vary from 4 to 11 $\mathrm{eV}$. The largest binding energies are found in the first two rows of the periodic table $(\mathrm{H}$ to $\mathrm{Ne})$. Up to $\mathrm{Z}_{t}=4$ the outer shells are energetically clearly separated from the inner shells and the proton induced stopping-power maximum is determined by the valence band only. For heavier targets the situation changes and the large number of electrons in bands energetically somewhat below the valence band has a significant influence. Thus, the averaged binding energy for heavy targets is enhanced. Furthermore, the mean electron velocity in the valence band of heavy targets is somewhat larger compared to light targets due to the acceleration in the vicinity of the screened target nuclei.

Both effects, binding-energy blending and valence-electron acceleration, are similar important as the valence binding-energies and lead on the average to increased energies of the stopping maximum for heavy targets. With these arguments it becomes also clear why Li has a very low stopping power maximum at about $40 \mathrm{keV}$. This metal has slow and weakly bound conduction electrons well separated from the K-shell binding energy. For a He target the maximum is found at $80 \mathrm{keV}$ and for heavy targets between 70 and $150 \mathrm{keV}$. This dependence on the $1^{\text {st }}$ binding energy and on the deeper level structure needs a detailed quantitative investigation and will not be further discussed here.

None of the above arguments explains the projectile nuclear charge de- 


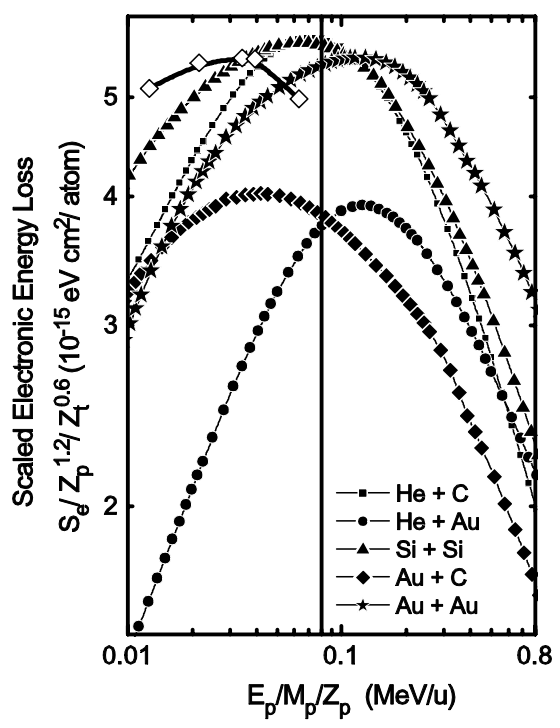

Figure 5: Scaled electronic energy loss for a few collision systems scattered among the periodic table as a function of a scaled projectile energy. Fit results for the electronic energy loss $\mathrm{S}_{e}$ are used representative for the experimental data [42].

pendence. Hence, we have performed ab-initio energy-loss calculations for a carbon target using the unitarized convolution approximation (UCA) $[33,34]$ to uncover the physical origin of the $\mathrm{Z}_{p}$ dependence. One example of theoretical results for $\mathrm{Au}+\mathrm{C}$ is shown in Fig. 5 as a thick solid curve with open diamonds. As an input, we have used numerical oscillator strengths for each target shell. Furthermore, a chargestate distribution centered around the mean projectile charge-state defined by the above formulas was used including shell effects. We have additionally computed the electron-loss contribution to the stopping power using a single oscillator strength per projectile shell. Previuosly, we have shown that the uncertainty of such a procedure is $<10 \%$ for heavy ions, even with less accurate charge-state formulas [43].

We find stopping-power maxima at $124 \mathrm{keV} / \mathrm{u}$ for $\mathrm{He}+\mathrm{C}$ and at $2.8 \mathrm{MeV} / \mathrm{u}$ for $\mathrm{Au}+\mathrm{C}$ (without $\mathrm{Z}_{p}$ scaling). The corresponding SRIM values are 144 $\mathrm{keV} / \mathrm{u}$ and $3.32 \mathrm{MeV} / \mathrm{u}$ respectively. For He ions the SRIM values appear to be more reliable, since there exists a huge amount of data points for the fitting procedure [44] and on the other hand our ab-initio code does not account for details of the valence-band structure. The situation is reverse for heavy ions. For the absolute values we find deviations of only about $7 \%$, slightly outside the experimental errors, from the data by $\mathrm{H}$. Geissel et al [20] for $\mathrm{Xe}, \mathrm{Pb}$ and $\mathrm{U}$ ions at $5 \mathrm{MeV} / \mathrm{u}$. These heavy-ion data are underestimated in SRIM by 15 to $25 \%$, with a maximum deviation for $\mathrm{Pb}$. Thus, our UCA code yields accurate absolute values and at least a reasonable $\mathrm{Z}_{p}$ dependence of the stopping maximum, when the target-shell structure, the projectile-charge distribution, projectile screening and the non-perturbative Bloch term are included in the calculation.

We have performed reference calculations also in 1st order perturbation theory (without the Bloch term) and without consideration of the electron loss. The result is $160 \mathrm{keV} / \mathrm{u}$, instead of $2.8 \mathrm{MeV} / \mathrm{u}$ when performing the full calculation. Consideration of electron loss would shift this perturbationtheory value further down in energy. Thus, the Bloch term is vital to the explanation of the energy scaling in Fig. 5. A full calculation for a fixed high projectile charge-state $\left(\mathrm{Au}^{48+}\right)$ shows the influence of the Bloch term very clear. High energy losses are strongly suppressed in the corresponding results and there is a very broad stopping power maximum centered around 670 $\mathrm{keV} / \mathrm{u}$. This, however, is still far below 
2.8 MeV/u.

Thus, we need the Bloch term as well as the projectile-charge variation to explain the linear scaling in $\mathrm{Z}_{p}$. For strong perturbations (slow heavy ions) the Bloch term leads to a strong suppression of the ionization probabilities, specifically at small impact parameters $[45,33]$. This flattens the stopping-power curves below a few $\mathrm{MeV} / \mathrm{u}$ and finally the charge-state variation determines the resulting position of the stopping-power maximum. An analysis of Eqs. 2 to 5 suggests that the scaling factor should be about $\mathrm{Z}_{p}^{0.92}$ if the energy dependence of the charge state alone would determine the stopping-power maximum. The stopping power maxima in Fig. 5 correspond to velocity-scaling parameters around $x=0.8$ or $\bar{q}^{2} \approx 0.5 Z_{p}^{2}$ in Fig. 4 , where the projectile charges are steeply increasing. In fact, we have also plotted the SRIM energy losses versus $x$ from Eq. 2, but the scatter of the corresponding stopping curves is comparable to the one in Fig. 5. This scatter, however, is consistent with the uncorrected target dependencies discussed above.

\section{Electron troscopy}

The investigation of projectile quantities such as scattering or energy loss may only yield information on the prompt reaction of the solid. Delayed emitted 'particles', however, may carry information on the track evolution. Here we will concentrate only on ejected electrons. Dependent on the material and on the investigated emission process, electrons are probes of the first $10^{-18}$ to $10^{-14} \mathrm{~s}$ of track formation and energy dissipation. Examples of such snapshots of the electronic track evolution are given below.
The experiments have been performed with highly charged particles at velocities of 6 to $13 \%$ the speed of light (at 1.78 to $8 \mathrm{MeV} / \mathrm{u}$ ). In most cases the ions were delivered by the heavy-ion cyclotron of the IonenstrahlLabor (ISL) at the Hahn-MeitnerInstitut Berlin. The only exception are the $8 \mathrm{MeV} / \mathrm{u} \mathrm{U}^{68+}$ data in Fig. 6 and one data point in Fig. 7 taken at the super-HILAC accelerator of the Berkeley National laboratory [46]. The Berlin setup is described in detail in ref.[47] and thus only a very brief explanation shall be given here.

The heavy-ion beam of 100 to 500 $\mathrm{nA}$ is focussed to a spot size of $2 \times 2 \mathrm{~mm}$ at the target (normal incidence) inside an ultra-high vacuum (UHV) chamber. Inside this doubly magnetically shielded scattering chamber (reduction of the earth magnetic field by a factor of 130) a high-resolution electrostatic electron spectrometer $(\Delta E / E=0.1 \ldots$ $1 \%)$ rotatable around the target center is operated. A stripper foil inside a doubly differential pumping stage directly in front of the main chamber yields a quasi-equilibrium charge-state distribution. This method was applied for nearly all ions and thus, there is no influence of non-equilibrium charge states and the corresponding reduction of track effects. In Figs. 79 , however, we also present data for the non-equilibrium charge-state ions $1.78 \mathrm{MeV} / \mathrm{u} \mathrm{Xe}^{15+}$ and $3.04 \mathrm{MeV} / \mathrm{u}$ $\mathrm{Au}^{30+}$ in amorphous Si. In this case, we estimate that about 0.5 projectile electrons will be stripped off within the first layer (corresponding to the mean free escape depth [48] for the investigated Si-L Auger lines), leading to a slightly enhanced mean projectile charge-state. This enhancement is so small that it has been neglected in the following.

Experimental results are presented for amorphous targets of graphitelike $\mathrm{C}$ (a-C, with an atomic hydro- 
gen fraction of $<10 \%$ ), Si (a-Si) and polypropylene $\left(\mathrm{PP}, \mathrm{C}_{3} \mathrm{H}_{6}\right)$. Thus, we will be able to detect differences in the behavior of metals, semiconductors and insulators. The surfaces of the boron doped Si (111) samples $(\rho<1$ $\Omega \mathrm{cm})$ were initially chemically etched and sputter cleaned with $2.5 \mathrm{keV} \mathrm{Ar}$ ions. Annealing at $850^{\circ} \mathrm{C}$ was used to outgas the Ar contaminants, thereby recrystallizing the sample. Afterwards an amorphized surface layer down to a depths of about $14 \mathrm{~nm}$ was produced by irradiation with $5 \mathrm{keV} \mathrm{Ar}$ ions for a few minutes. All Si experiments have been performed under UHV conditions at residual gas pressures far below $10^{-9}$ mbar (dominated by $\mathrm{H}_{2}$ ). The atomically clean target surfaces (all-over contaminations $<3$ atom\%) were characterized by Augerelectron spectroscopy before and after the ion-irradiation cycles that were lasting a few hours, dependent on the actual vacuum conditions. In between, sputtering and amorphization with 5 $\mathrm{keV}$ Ar ions for up to 20 minutes was used to clean the samples.

All experiments with PP and most experiments with amorphous $\mathrm{C}$ (a-C) were performed with less sophisticated experimental setups $[38,49]$ at residual pressures of $10^{-6}$ mbar, dominated by $\mathrm{H}_{2} \mathrm{O}$, without cleaning and annealing possibilities. a-C is quite inert to oxidation and furthermore, fast heavy-ion beams lead to an electronic desorption of surface contaminants. Thus, constant surface oxygen contaminations of typically a few atom $\%$ were observed during the experiments. All results presented in this work are believed to be not affected by this coverage, since the coverage was stable after some minutes of heavy-ion irradiation. Moreover, the mean free electronescape depth at an electron-emission angle of $135^{\circ} \mathrm{C}$ corresponds to 4 layers in a-C (at an ejection energy of 270 eV) [48] reducing the relative influence of a surface coverage.

It is emphasized that $\mathrm{Si}$ is oxidizing rapidly under such vacuum conditions and at a typical energy of $90 \mathrm{eV}$ the Auger signal is dominated by the upper two surface layers only [48]. Thus, for $\mathrm{Si}$ and for most other materials UHV conditions are absolutely necessary. Nevertheless, we have performed test experiments with slightly annealed diamond-like amorphous carbon (DLC), with sputtercleaned graphite-like a-C as well as with cleaved and annealed crystalline graphite. Within the experimental uncertainty these UHV results for carbon agree perfectly with our previous data [50]. Thus, in this work we do not distinguish the UHV results for a-C from the majority of the data points.

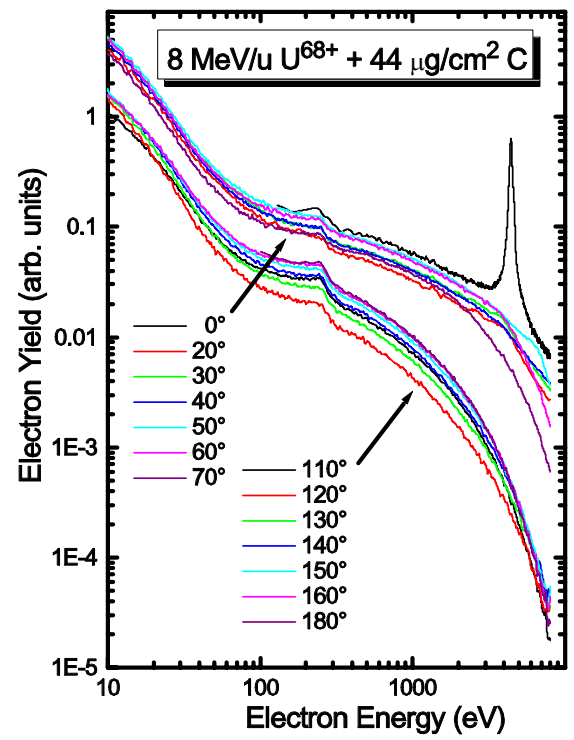

Figure 6: Experimental electronenergy distributions for $8 \mathrm{MeV} / \mathrm{u} \mathrm{U}^{68+}$ ions penetrating a thin amorphous carbon foil. The electron ejection-angle was varied between 0 and 180 degrees with respect to the incident beam direction. 
Fig. 6 displays electron energy spectra for emission angles between $0^{\circ}$ and $180^{\circ}$ with respect to the ion-beam direction for $8 \mathrm{MeV} / \mathrm{u} \mathrm{U}$ ions close to their equilibrium charge state in amorphous carbon. The measurements were performed with a low energy resolution of $7 \%$ to achieve high count rates at all energies. This plot shows the most important ejection mechanisms that can be investigated using electron spectroscopy.

There is a smooth continuously falling background visible at all angles. These electrons at energies between about $70 \mathrm{eV}$ and several $\mathrm{keV}$ are the so-called $\delta$-electrons. They are produced in violent binary collisions of the projectile ion with target electrons. At an ejection angle of $50^{\circ}$ there is broad bump visible in the spectrum at an energy of about $7 \mathrm{keV}$. This bump corresponds to the electron energy

$$
E_{e}^{\text {binary }}=\frac{4 E_{p} m_{e}}{M_{p}} \cos ^{2}\left(\theta_{e}\right)
$$

that reflects binary collisions between a heavy projectile and a free electron initially at rest. At larger angles this bump is too broad to be visible and at lower angles the binary-encounter energy exceeds the maximum experimental energy of $8 \mathrm{keV}$. Transport calculations show that the yield of fast electrons emitted in backward directions is due to the same binary-encounter processes followed by multiple angular scattering inside the solid [46]. At energies below about $70 \mathrm{eV}$ there is a change of the spectral slope of the background due to the so-called softcollision electrons or true secondary electrons. This structure belongs to a peak centered at an energy of a few $\mathrm{eV}$ and consists mainly of the slow electrons resulting from electron-collision cascades near the surface.

The most pronounced structure in Fig. 6 is found at $0^{\circ}$ for a detection energy of $4400 \mathrm{eV}$. This is the convoyelectron peak and its electron-velocity vector corresponds to the projectile velocity. This peak is due to electron capture to the projectile continuum (ECC) and due to electron-loss to continuum (ELC) processes. Its intensity is strongly influenced by the long ranged Coulomb force of the projectile. Convoy electrons suffer collisions with target electrons and are steadily attracted by the projectile Coulomb potential, giving rise to a random walk in the projectile reference frame. The energy position of the convoy peak is slightly sensitive to surface potentials as well as to the ion-track potential [52]. However, so far it was not possible to extract quantitative track properties from such measurements because of the complicated random-walk processes. At angles between 20 and $40^{\circ}$ one may recognize weak bumps at an energy of about $4000 \mathrm{eV}$. These bumps are due to ELC processes with somewhat larger energy transfers. Inside the solid electrons are liberated from the projectile. They start at about the projectile velocity and loose a certain amount of energy until they reach the surface.

At an electron energy around 270 $\mathrm{eV}$ there are peak structures superimposed on the continuous $\delta$-electron background in all of the spectra. These peaks are the carbon KVV Auger structures (here $\mathrm{K}$ stands for a $\mathrm{K}$ vacancy and each $\mathrm{V}$ stands for a valenceband electron that is active during the Auger decay). Auger peaks are due to the delayed two-electron decay of inner-shell vacancies. In the case of carbon a $\mathrm{K}$ vacancy is filled by a valence electron and another valence electron is ejected. The residual electron-electron interaction is responsible for this transition and the Auger transition rates for $\mathrm{C}-\mathrm{KVV}$ and $\mathrm{Si}$ LVV exceed the x-ray transition rates by about a factor of 1000 [53]. From 
such spectra measured at high resolution one may determine the degree of inner shell ionization from an analysis of the multiple peak structure (see section 4). Furthermore, it is possible to determine the ion-track potential from an Auger peak-shift (for a detailed discussion see section 5) and the electron temperature in the valence band is related to the high energy slope of the peak (see section 6).

It is well known that fast electrons are predominantly ejected into forward directions (see also Fig. 6). This is a direct consequence of two-body collisions dynamics. As has been shown in previous investigations for carbon targets [51, 46, 38], these fast electrons are so intense that the number of inner-shell vacancies resulting from cascade collisions inside the target is comparable to the direct innershell ionization by the projectile ion. Thus, Auger electrons that are emitted into the forward hemisphere (from the ion-exit surface) in thin-film experiments are to a large extend produced far away from the track by secondary electron-collision cascades. Contrary, Auger electrons ejected in backward directions are mainly induced directly by the projectile (in the central track region). Thus, in the following detailed analysis of track effects only data for a single fixed detection angle of $135^{\circ}$ with respect to the ion-beam direction is considered, corresponding to $45^{\circ}$ with respect to the surface normal.

For all data presented subsequently, electron energy-spectra have been taken not only for incident heavy ions. In addition reference and surfacecontrol spectra where also taken with electrons at similar incident velocities (1 and $2.7 \mathrm{keV}$ at an incidence angle of $\left.45^{\circ}\right)$. During the experiments, the electron beam $(\emptyset<1 \mathrm{~mm})$ is focussed at the centre of the ion irradiated spot with an uncertainty of about $\pm 0.5 \mathrm{~mm}$. Thus, surface coverages or ion induced phase transformations may be detected in between the ion runs.

\section{Multiple Ionization}

In this section we present results on the intensity of multiple inner-shell ionization of $\mathrm{C}$ and $\mathrm{Si}$. A single $\mathrm{K}$ vacancy in $\mathrm{a}-\mathrm{C}$ leads to the peaks at about $260 \mathrm{eV}$ in Fig. 6. Double $\mathrm{K}$ vacancies in a-C lead to a broader shoulder around $310 \mathrm{eV}$ (barely visible in the figure because of low energy resolution and low counting statistics in the double logarithmic plot). Auger lines due to multiple inner-shell vacancies decay in the same way as for a single vacancy. These structures, however, are always shifted to higher emission energies, since the reduced inner-shell screening leads to an increased innershell binding-energy. For the following discussion intensity data are extracted from integrated multiple Auger peakstructures after subtraction of the $\delta$ electron background and an iterative separation of the peaks belonging to different vacancy numbers.

It is emphasized that the vacancy production is part of the energy-loss processes. The typical time for creation of a $\mathrm{K}$ vacancy is about $2 \times 10^{-18}$ $\mathrm{s}$ at $5 \mathrm{MeV} / \mathrm{u}$. Thus, in comparison to all other important time scales the Auger intensities are effectively sensitive to the ion passage at time zero. They are also only sensitive to the center of the infra track, since the corresponding ionization probabilities drop drastically beyond impact parameters of $0.5 \AA$.

As the carbon Auger spectrum has already been introduced above, a short description of the Si Auger spectrum is still needed for the discussions below. The ion-induced target Auger spectrum of Si involves Auger structures at energies between 88 and 132 $\mathrm{eV}$ due to one up to four L-shell va- 
cancies in the $2 p$ shell $\left(2 p^{1} \mathrm{VV}, 2 p^{2} \mathrm{VV}\right.$, $2 \mathrm{p}^{3} \mathrm{VV}$ and $2 \mathrm{p}^{4} \mathrm{VV}$ ). Furthermore, a vacancy in the $2 \mathrm{~s}$ shell leads to fast $2 \mathrm{~s} 2 \mathrm{p}^{j} \mathrm{~V}$ Koster-Kronig transitions (intrashell Auger decay) at energies up to $42 \mathrm{eV}$, where one $2 \mathrm{p}$ electron (out of 6 -j) fills the 2 s-hole by transferring energy to a valence electron. In the following, line intensities for a-Si and a-C will be used to derive information on the initial degree of ionization inside the track.

Fig. 7 displays integrated Auger yields $\mathrm{Y}_{n}$ for $\mathrm{n}=1$ to 4 inner-shell vacancies of a-Si and a-C [54]. The sum of the data for each target is normalized to one. Results are plotted as function of the electronic perturbation parameter or interaction strength $\mathrm{P}=\left|q_{e f f}\right| / \mathrm{v}_{p}$, as it appears in quantum mechanical matrix elements for electronic excitations. The projectile velocity in units of the Bohr velocity $\left(2.19 \times 10^{6} \mathrm{~m} / \mathrm{s}\right)$ is denoted $\mathrm{v}_{p}$, and the effective charge $\mathrm{q}_{\text {eff }}$ is set equal to the mean incident particle charge state for projectiles in their charge-state equilibrium. Only for the non-equilibrium ions $1.78 \mathrm{MeV} / \mathrm{u} \mathrm{Xe}^{15+}\left(\mathrm{q}_{\text {eff }}=21\right)$, $3.04 \mathrm{MeV} / \mathrm{u} \mathrm{Au}^{30+}\left(\mathrm{q}_{\text {eff }}=38\right.$ ), and for $0.94 \mathrm{MeV} / \mathrm{u} \mathrm{S}^{6+}\left(\mathrm{q}_{\text {eff }}=9\right.$, displayed in Fig. 7), we have modified $q_{\text {eff }}$ considering the projectile electron-loss at the surface and the reduced projectile screening dependent on the involved projectile and target shell radii.

Furthermore, we have reanalyzed the $\mathrm{Si}$ spectra for electrons, protons and $\mathrm{S}^{6+}$ from the pioneering work by Schmidt et al. [55]. The results are shown as open symbols in the lower plot. It is noted that the spectra by Koyama et al. [56] have not been analyzed, since electron reference-spectra are missing. High-energy data at 8 $\mathrm{MeV} / \mathrm{u}$ by Caron et al. [57] for a$\mathrm{C}$ targets are included as well in the plot (open symbols). The error bars in Fig. 7 indicate all-over estimates of the uncertainty. In the figure we also have included theoretical results that are based on quantum mechanical ionization probabilities and classical transport theory as will be explained in the following.

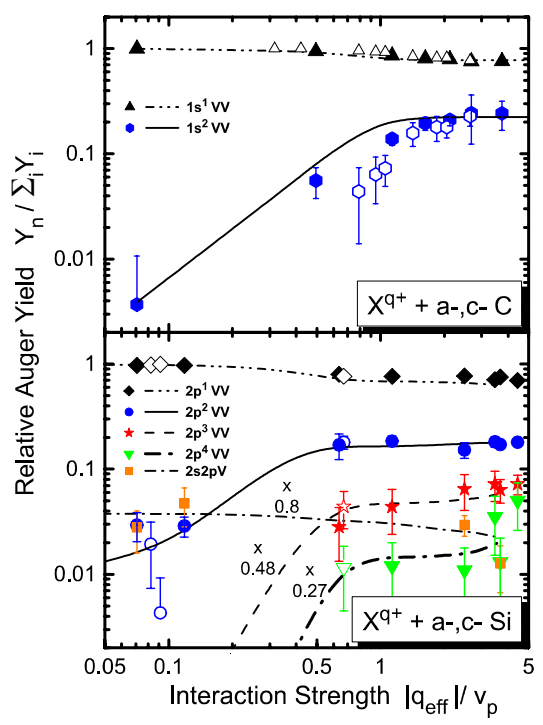

Figure 7: Normalized experimental (symbols) and theoretical (lines) Auger yields as function of the electronic interaction strength for incident electrons and heavy ions. The data in the upper plot are obtained for carbon and in the lower polt for silicon.

For $\mathrm{Si}$, the theoretical treatment is based on the Magnus approximation [31, 50] (including shake-off) for ionization of the $2 \mathrm{~s} 0,2 \mathrm{p} 0$, and $2 \mathrm{p} \pm 1$ states given by the Si Hartree-FockSlater potential. The resulting unitarized ionization probabilities for 5 $\mathrm{MeV} / \mathrm{u}$ have been calculated from total $1^{\text {st }}$-order Born ionization-probabilities $P_{B 1}^{i o n}\left(Z_{p}, b\right)$ according to

$$
P_{M 1}^{i o n}\left(Z_{p}, b\right)=\sin ^{2}\left(Z_{p} \sqrt{P_{B 1}^{i o n}(1, b)}\right)
$$

and converted into multiple-ionization cross sections using the (statistical) 
independent electron model (IEM). Auger cascades have also been considered as explained further below. The $2 \mathrm{pVV}$ Auger transition corresponds to a decay time of $15 \mathrm{fs}$, whereas the $2 \mathrm{~s} 2 \mathrm{pV}$ Koster-Kronig decay time is only $0.7 \mathrm{fs}[53,58,59]$. Using simple statistics we estimate that the KosterKronig decay is faster than the $2 \mathrm{p}^{n} \mathrm{VV}$ Auger transitions, even for a 4-fold ionized $2 \mathrm{p}$ shell $(\mathrm{n}=4)$. Thus, $2 \mathrm{~s}$ vacancies will lead to a $2 \mathrm{~s} 2 \mathrm{pV}$ transition, increasing the number of $2 \mathrm{p}$ holes from $n$ to $n+1$. Afterwards, the $2 \mathrm{p}^{n} \mathrm{VV}$ transitions will take place, leading to a remaining $(n-1)$-fold ionized $2 \mathrm{p}$ shell. In this way, a whole series of Auger electrons results from one multipleionization event. At this point the theoretical results would represent a quasi-atomic case.

Thus, corrections for the electron escape-depth and for $\delta$-electron cascades inside the solid have been applied. Fast $\delta$-electrons may produce single L-shell vacancies far away from the track. As we have estimated from our previous work for $\mathrm{C}$ targets $[51,49]$, considering the differences in backscattering yields and binding energies between $\mathrm{C}$ and $\mathrm{Si}$, the total Augerelectron yield in Si contains a $30 \%$ fraction due to these $\delta$-electron cascades and subsequent $2 \mathrm{p}^{1} \mathrm{VV}$ transitions.

Furthermore, transport calculations of the energy dependent electron escape-depths were performed including penetration of the surface barrier [60] with a focus on the high-energy behavior of the electron energy-loss spectrum for homogeneously distributed electron sources at the experimental line positions. The emitted electron intensities have been integrated in the same way as for the experimental data. The resulting emission weightfactors are $0.50(2 \mathrm{~s} 2 \mathrm{pV}), 1\left(2 \mathrm{p}^{1} \mathrm{VV}\right)$, $1.24\left(2 \mathrm{p}^{2} \mathrm{VV}\right), 1.57\left(2 \mathrm{p}^{3} \mathrm{VV}\right)$, and 1.93 $\left(2 \mathrm{p}^{4} \mathrm{VV}\right)$ for the different Auger lines. The final yield curves for $2 p^{n} V V$ are proportional to $P^{2 n-2}$ for small values of the perturbation parameter $\mathrm{P}$ (perturbation limit of the IEM) and for large values of $\mathrm{P}$ they nearly reach a plateau. Exceptions are the curves for $2 p^{2} V V$ transitions, where an offset due to shake-off processes is included, and for $2 \mathrm{~s} 2 \mathrm{pV}$ transitions which are both dominated by single ionization in the case of small P.

Comparison of the experimental and theoretical results for $\mathrm{Si} 2 p^{1} V V$ and $\mathrm{Si} 2 p^{2} V V$ shows reasonable agreement. However, discrepancies become significant for the less intense lines and reach a factor of 0.27 for the $2 p^{4} V V$ decay (note that we heave observed an indication of 5 -fold $2 \mathrm{p}$ ionization by $\mathrm{Au}$ projectile). This reflects a well-known behavior of the IEM which neglects the dependence of the ionization potential on the degree of ionization. The flat behavior of the curves for high values of $\mathrm{P}$ is related to the Magnus prediction of an ionization probability close to $100 \%$ at small impact parameters. Accounting for the deviations between experiment and theory, there will be about $55 \%$ L-shell ionization and complete valence-band ionization inside a track diameter of about $1.6 \AA$ for $\mathrm{U}$ projectiles at $5 \mathrm{MeV} / \mathrm{u}$. For the a$\mathrm{C}$ target the theoretical treatment is very similar and comparison with the experimental data shows a good overall agreement, especially for large perturbations. Thus, there is complete (6-fold) ionization of $\mathrm{C}$ for heavy ions with $Z_{p}>30$ at $5 \mathrm{MeV} / \mathrm{u}$.

The above discussion shows, there is an enormous high degree of ionization directly after the interaction of the projectile with the target-electron system. The influence of this initial stage of the track evolution on the electron dynamics at much longer time scales is investigated in the next sections. Specifically one may ask the question, whether the liberated electrons do return and screen the positive charges in 
the center of the ion track before the Auger decay takes place.

\section{Ion-Track Potential and Coulomb Explo- sion}

Strong ionization of atoms inside the ion track leads to a cylinder of positive charges and a resulting positive ion-track potential. It is possible to detect this potential if the charge neutralization is slow enough. The iontrack potential does not act on emitted photons (no influence on x-ray line structures), but it decelerates emitted electrons, which have to overcome the corresponding potential barrier. Line structures in the electron spectrum, either the convoy-electron peak or Auger lines, may be used to determine this deceleration. In fact, a deceleration of convoy electrons compared to the projectile speed (see the explanation of Fig. 6) as well as an Auger electrondeceleration has been found for the insulators polypropylene $\left(\mathrm{PP}, \mathrm{C}_{3} \mathrm{H}_{6}\right)$ and also mylar $[49,52,61]$. In the following, Auger results for PP will be compared to recent data for a-Si $[47,54]$.

Similar to the previous section the Auger line-shift is sensitive to the potential in the center of the track, since the residual electron-electron interaction is short ranged. Contrary to the previous section, however, the lineshift is determined by the solid-state environment during the statistically delayed Auger decay process. Typical Auger decay times are $11 \mathrm{fs}\left(11 \times 10^{-15}\right.$ s) for carbon $\mathrm{K}^{1} \mathrm{VV}$ and 15 fs for silicon $2 \mathrm{p}^{1} \mathrm{VV}$ lines. For multiple vacancy lines $\left(\mathrm{C}-\mathrm{K}^{2} \mathrm{VV}\right.$ and also Si- $2 \mathrm{p}^{2} \mathrm{VV}$, Si$\left.2 \mathrm{p}^{3} \mathrm{VV}, \ldots\right)$ the decay times are significantly reduced. Hence, an analysis of the Auger line positions for different vacancy states yields snapshots of the potential evolution inside the track for different times.

Fig. 8 displays Auger energy reductions, determined relative to a reference value, as a function of the perturbation parameter $\mathrm{P}=\left|q_{e f f}\right| / \mathrm{v}_{p}$. Note that $q$ has been used here instead of $q_{\text {eff }}$ as in Fig. 7, since inner-shell excitations are of minor importance for the track potential, and valenceband excitations are dominated by the projectile-electron interactions at large impact parameters. Peak positions corresponding to fast electron- or proton-induced spectra serve as a zero reference-value for the $\mathrm{Si}$ values in this plot. This choice shall be explained in detail before the results of Fig. 8 are discussed. For the insulator PP the situation is much more complicated, since there is a strong macroscopic charging during electron irradiation, which vanishes nearly completely during heavyion irradiation. Therefore, other materials namely amorphous carbon and $\left[\mathrm{C}_{2.1} \mathrm{H}_{0.6}\right]_{n}$ instead of $\left[\mathrm{C}_{3} \mathrm{H}_{6}\right]_{n}$ had to be used for the energy reference. Both reference materials show no significant Auger shift. For details of this method the reader is referred to refs. [49, 61 , 50].

For light charged-particle induced ionization as well as for non-resonant $\mathrm{x}$-ray induced photo ionization of inner shells, there are only minor dependencies of the line shape or position on the primary excitation. Auger lines as well as x-ray emission-lines show the so-called dynamic initial-state screening effects. Typically, these are weak secondary modifications of the initialstate population triggered by the dynamics of the ionization process. Quite often shake-up and shake-off processes determine the dynamic initial-state screening for weakly interacting particles such as individual photons or electrons. As an example, one may notice that the relative $2 \mathrm{p}^{2} \mathrm{VV}$ ionization yield in Fig. 1 approaches a finite value of $0.8 \%$ for fast incident electrons and 


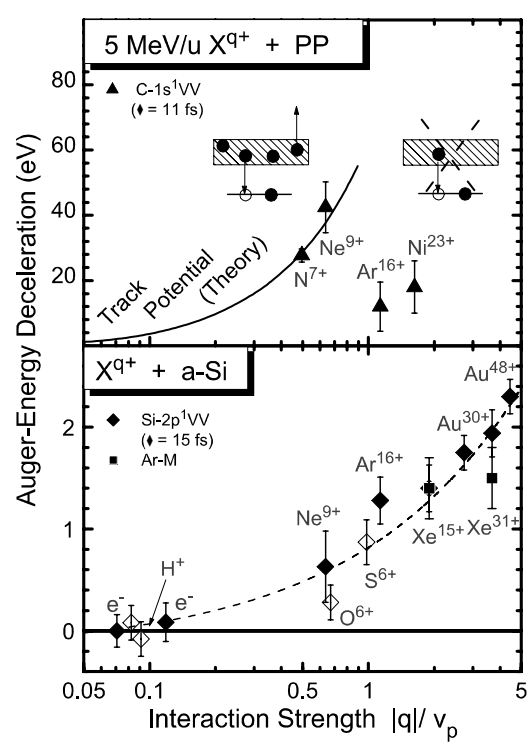

Figure 8: Auger energy reduction vs. $|q| / v_{p}$. The upper plot shows the carbon KVV Auger-energy shift obtained for polypropylene (PP) samples in comparison to track theory. The lower plot shows LVV energy shifts for amorphous Si.

protons (shake-off limit at $\mathrm{P}<<0.1$ ) and reaches $20 \%$ for swift heavy ions at $5 \mathrm{MeV} / \mathrm{u}$ (dominated by $2^{\text {nd }}$ order two-step mechanisms).

In Fig. 8, data are presented for the carbon $1 \mathrm{~s}^{1} \mathrm{VV}$ Auger peak of polypropylene $[49,61,50]$ and for two Auger peaks corresponding to the $\mathrm{Si}$ target. The $2 \mathrm{p}^{1} \mathrm{VV}$ peak corresponds to the main line of $\mathrm{Si}$ (see Fig. 7) and the Ar-M peak corresponds to a weak Ar contamination after sputter cleaning that has been detected in two of the experiments. The open symbols for aSi have been extracted from the spectra by Schmidt et al [55] for electrons, $\mathrm{H}^{+}, \mathrm{O}^{6+}$ and $\mathrm{S}^{6+}$. The dashed curve for $\mathrm{Si}$ is shown to guide the eye.

As mentioned above $\delta$-electron induced vacancies at the ion-entrance surface constitute a fraction of only about $7 \%$ for a thick a-C target. They may, however, amount to $30 \%$ for the a-Si target because of enhanced backscattering yields for higher values of $\mathrm{Z}_{t}$. It is noted that we have not performed a correction for the influence of $\delta$-electron cascades on the silicon $2 \mathrm{p}^{1} \mathrm{VV}$ line position. This awaits a more detailed understanding of the line shape.

The carbon peak-shifts increase with the interaction strength $\mathrm{P}$ up to a value of value of about $42 \mathrm{eV}$ at $\mathrm{P}=0.6$. For larger interaction strengths the ion-track potential seems to drop to a much lower value. The initial rise of the potential is consistent with the solid curve, a computed ion-track potential [49]. The model is a combination of classical-trajectory Monte Carlo calculations for the electron motion in the field of the projectile ion under the assumption of a continuousslowing down behavior inside the solid. Thus, the electron displacements are computed as a function of time and the sum over all positive and negative Coulomb potentials yields a screened track potential dependent on the time and on the distance to the center of the track.

The good agreement between experimental data for $\mathrm{N}$ and $\mathrm{Ne}$ ions and the model results is taken as evidence for a strong suppression of track neutralization. The values for $\mathrm{Ar}$ and $\mathrm{Ni}$ ions, however, are much lower. This is also consistent with the model as for $\mathrm{P}>0.8$ there are less than two valence electrons per atom in the center of the track. Thus, Auger decay is impossible and the Auger clock stops. Only at a later stage during the slow neutralization process there will be two valence electrons or more, which is the necessary condition for the Auger transition. Consequently, for very heavy ions the Auger decay takes place in an electronically relaxed environment and the resulting Auger shift must be far below 
the value of the initial track potential.

Thus, all PP results in Fig. 8 appear to be completely consistent with a long-lived strong ion-track potential. Quantitative estimates indicate that the track life-time is $>1.5 \times 10^{-14} s$. Thus, for heavy ions, the ion-track potential in polypropylene is strong enough and survives long enough to allow at least for a partial Coulomb explosion of the protons in the polymer. Indications for a strong erosion due to Coulomb explosion [50] and for a related small energy shift of ejected hydrogen ions [62] have been found. For other carbon structures, such as amorphous diamond-like and graphite-like carbon as well as crystalline graphite, energy shifts are below the corresponding experimental uncertainty of $2 \mathrm{eV}$. Thus, neutralization is much faster in these materials and Coulomb explosion cannot take place.

The silicon peak-shifts in Fig. 8 increase monotonically with the interaction strength $\mathrm{P}$ and reach about $2.3 \mathrm{eV}$ at $\mathrm{P}=4.4$. Furthermore, the shifts are very similar for the different $\mathrm{Si}$ Auger lines (see ref. [54] for preliminary evaluations of other Si lines). Macroscopic charging of the B-doped $\mathrm{Si}$ samples can be excluded for the observed effect, since no indication of a peak shift could be found for incident electrons at different beam currents. Materials modification can be excluded as well, since the electron reference-spectra, taken before and after the ion-measurement cycles, are identical to within an uncertainty of $\pm 0.15 \mathrm{eV}$

Thus, we also attribute the measured shift for Si to the ion-track potential induced by a local reduction of the electron density as a result of ionization in the center of the track. Auger electrons are decelerated when leaving such a positively charged region. From our previous investigations of this effect for polypropylene (PP, see upper part of Fig. 8) and mylar $[49,61,50]$, we estimate that the initial track potential directly after the interaction with the projectile should be about $250 \mathrm{~V}$ for $\mathrm{Xe}^{31+}$ ions (at $|q| / \mathrm{v}_{p}=3.7$ ) and nearly independent of the material. Thus, the measured shift of only $2.3 \mathrm{eV}$ in silicon is strongly influenced by the time dependent electronic neutralization of the track. Assuming an exponential decay, however, a time dependence of the potential should show up in the different Si Auger line-shifts that cover effective decay times from about 1 to 17 fs. Hence, the major part of the neutralization is very rapid $(<1 \mathrm{fs})$ and the measured shift seems to be related to a very slow and weak component of the ion-track potential that might be due to long-lived traps (trapped excitons and populated defect states) in the amorphous material.

Significant ion-track potentials seem to be exclusively related to either insulators or semi-conductors. No significant line shifts have been found for metals so far. Correspondingly, a Coulomb explosion can be excluded for metals as well as for a-Si (because of the small value of the track potential). Thus, materials modification processes in metals call for a different energy conversion mechanism as will be discussed in the following section.

\section{Electron Temper- atures, Thermal Spike and Lattice Relaxation}

In this section we will analyze implications of the thermal spike and lattice relaxation models as a result of highly excited track cores after neutralization. Experimental data for the electron temperature after neutralization, the main ingredient of both mod- 
els, are presented for the first time for a-Si. Similar as in section 5, the temperature data are sensitive to the center of the track and to the Auger decay times of a few femtoseconds.

So far, we have investigated the intensity (see Fig. 7) and the shift of Auger lines (see Fig. 8). But the slope of the Auger lines carries information about the degree of excitation of the valence band during the vacancy decay. The high energy shoulder of the Auger structures reflects a convolution of the populated density of states near the Fermi level [63, 66, 67]. As described in detail in previous publications on a-C [64] the line widths increase with increasing projectile charge-state related to an increasing electron temperature. The data evaluation is based on a comparison with Auger spectra for incident electrons, as a reference for the electron transport properties. First we fit these spectra using a simple model for electron transport [65] that includes the density of states, assuming that the corresponding electron temperature in the valence band is nearly zero. Electron temperatures are than extracted from fits to ion induced spectra, by variation of the Fermi-Dirac distribution and by keeping all other transport properties fixed.

Fig. 9 displays experimental electron temperatures vs. interaction strengths $\mathrm{P}=|q| / \mathrm{v}_{p}$ for the Si $2 \mathrm{p}^{1} \mathrm{VV}$ Auger decay. The error bars indicate the overall uncertainty of the current evaluation dominated by the curvefitting procedure. The measured temperature is monotonically and slowly increasing with $\mathrm{P}$ and reaches about $15000 \mathrm{~K}$ for $3 \mathrm{MeV} / \mathrm{u} \mathrm{Au}^{48+}$ ions. It is emphasized, however, that these data should be taken as preliminary since $\delta$ electron cascades have not been considered in the evaluation. The $\delta$-electron cascades are expected to reduce the broadening of the measured Auger lines, since they contribute "cold" sec-

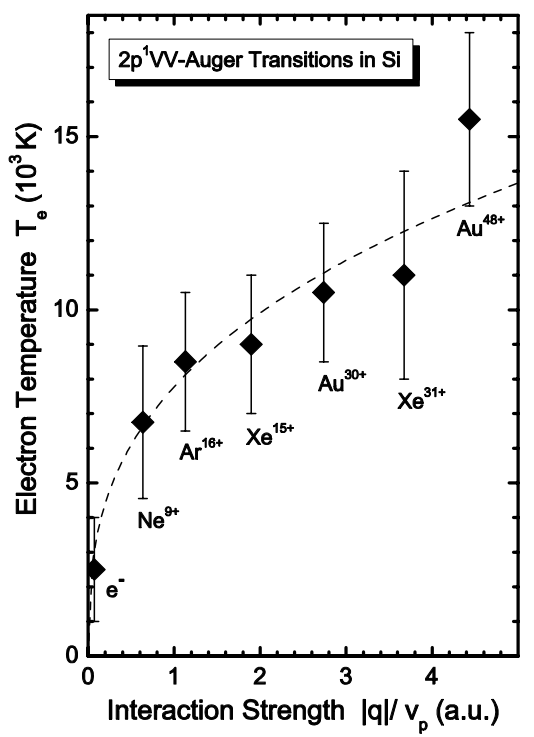

Figure 9: Electron temperature as determined from the broadening of the dominant Auger line of amorphous Si for different projectiles at 1.8 to 5 $\mathrm{MeV} / \mathrm{u}$. The dashed curve is shown to guide the eye.

ondary Auger electrons from regions that are far away from the track. Correspondingly, we expect that the analyzed temperatures will rise by about $30 \%$ when this effect is included.

Furthermore, it is possible to improve the accuracy of the fit significantly when improved partial density of states (PDOS) are considered. An analysis of the PDOS [66, 67 used in this work in comparison to experimental data for UV and $\mathrm{x}$ ray photo-electron emission (UPS and XPS), high resolution soft x-ray emission spectroscopy (XES), x-ray absorption near-edge spectroscopy (XANES), and bremsstrahlung isochromate spectroscopy (BIS or inverse XPS) has clearly revealed inconsistencies between experiment and theory. Thus, 
a more involved analysis of the PDOS for $\mathrm{Si}$ is necessary and more recent accurate theoretical results [68] should be used as a guide-line to determine a more reliable PDOS from the experimental data.

Previous data for graphite-like amorphous carbon [64] and other carbon modifications [50] did not show such problems, since the corresponding PDOS [63] is broad due to the extremely high Fermi energy of a-C and graphite. The results for $\mathrm{C}$ show a similar tendency as a function of $\mathrm{P}$ as the ones displayed in Fig. 9. However, for high projectile energy-losses electron temperatures of about $80000 \mathrm{~K}$ are reached. These have been compared with two thermal-spike models. Comparison with results of the free-electron code by Toulemonde and coworkers [12] show up to an order of magnitude deviation for light ions. This failure of simple thermal-spike models and the corresponding data from two experimental groups are presented and discussed in a review article by Rothard within this topical issue. It should be noted, however, that improved theoretical treatments accounting for the DOS of a-C in the computation of the electronic heat capacity and the electronic thermal conductivity agree to within $35 \%$ with the experimental data.

Accounting for the influence of $\delta$ electron cascades in $\mathrm{Si}$, electron temperatures will probably exceed 20000 $\mathrm{K}$. This might be high enough to trigger phase transitions in $\mathrm{Si}$ if the electron-phonon coupling is strong. At these high temperatures, however, about $12 \%$ of the valence electrons are excited into higher lying surface states and continuum state of the bulk. Under such conditions a spontaneous lattice-relaxation, driven by inter-atomic non-equilibrium potentials, can also not be excluded as a materials modification mechanism [8].

\section{Conclusions}

The energy dissipation due to fast heavy ions in matter is investigated with special attention on track effects at the very center of an ion path on a sub-picosecond time scale. Basic energy-transfer mechanisms and electronic relaxation processes are reviewed in the light of recent experimental and theoretical developments.

From the view point of a swift heavy projectile, its speed and its charge state and the resulting energy transfer to the target are the most important parameters that determine track-production processes. Ion charge-states in matter can now be predicted with high precision on the basis of a semi-empirical fit to the existing data. Projectile-shell effects, a target dependence of the mean chargestate and now also resonance effects have been identified with high significance. Especially for surface experiments, but also for thin-film experiments, non-equilibrium charge-states have to be considered if the mean energy loss shall be a meaningful parameter for the analysis of experimental data [34].

It is shown that the energy loss of fast ions is reasonably well understood. A non-trivial explanation for the approximate $\mathrm{Z}_{p}$ scaling of the energy at the stopping-power maximum has been found in this work. The deviations between experimental stopping powers and theoretical ab-initio results are currently below $10 \%$ for fast heavy ions. It even seems that current abinitio stopping powers $[33,34,35,36$, 37] are more accurate for fast heavy ions than the most recent version of the well-known semi-empirical SRIM tabulations [42]. Consistent inclusion of the accurate mean charge states presented here, of electronic polarization effects (the so-called Barkas term) [35, 69] and of excited projectile states $[70,36,71$, 
$72,73]$ is expected to reduce the theoretical uncertainties by another factor of 3, an important goal for precision ion-beam analysis. Finally one would be limited by dynamic meanfield effects and electron-correlation effects which are difficult to include in a many-electron treatment. Already today, however, the prediction of impactparameter dependent non-equilibrium or equilibrium energy-losses appears to be no significant source of uncertainty for the explanation of track effects (at $\left.E_{p} / M_{p}>>100 \mathrm{keV} / u\right)$.

Reasonable agreement is also found in a comparison of experimental and theoretical probabilities for multiple inner-shell ionization. For very heavy ions these results indicate that the center of the track is extremely strong ionized. For light targets such as carbon this even means complete ionization of all target electrons. Thus, after the ion passage the electronic structure inside a track is far away from equilibrium. The track volume is transformed into a highly charged column with hot electrons surrounding the ion path.

Hence, the main unsolved question concerning materials modifications by fast ions is 'how are such strong electronic excitations converted into atomic motion ?'. In order get some first answers to this question, high resolution in-situ electron-spectroscopy is applied to swift heavy-ion-solid interactions. Note that the possibilities of high resolution target x-ray spectroscopy have not even been partially explored until today.

In this work, we have presented and discussed results of target electronspectroscopy for the three materials amorphous graphite-like carbon (a-C), polypropylene ( $\left.\mathrm{PP},\left[\mathrm{C}_{3} \mathrm{H}_{6}\right]_{n}\right)$, and amorphous silicon (a-Si). Some published results exist also on crystalline graphite (HOPG) and amorphous diamond-like carbon (DLC) [50], on mylar [61] as well as prelimi- nary data on $\mathrm{Si}(111)$ with a $7 \times 7$ reconstruction [54]. On the basis of work in progress, we also do have further information on thin oxygen-implanted insulator films of $\mathrm{Al}_{2} \mathrm{O}_{3}$ and $\mathrm{BeO}$ [74] as well as on the pure metals $\mathrm{Be}$ and $\mathrm{Al}$ and a few metallic glasses [75].

The behavior of all these materials may be characterized in terms of two electronic properties that determine the atomic evolution of ion tracks

\section{- ion-track potential}

For the insulators polypropylene and mylar we have found high ion-track potentials and extremely high electronic sputtering yields with a threshold behavior. Both facts are strong indications for the Coulomb explosion mechanism, a mutual repulsion of highly ionized atoms. For the semiconductor a-Si a small positive potential is found, but it is definitely to weak to lead to a Coulomb explosion. For the insulating thin oxide films, however, there seems to be no significant ion-track potential according to a preliminary analysis. To within uncertainties of $\pm 0.3 \mathrm{eV}$ to $\pm 1 \mathrm{eV}$, all other investigated materials do also not show indications for an ion-track potential. Coulomb explosion can definitely be ruled out for these solids. Thus, some defect rich insulators or semiconductors with non-polar bonds seem to favor track potentials, but details remain unclear at the moment.

\section{- electron temperature}

For the all materials where electron reference spectra could be obtained we found a broadening of the ion induced Auger spectra. This broadening is related to high electron temperatures in a range of about $15000 \mathrm{~K}$ 
up to $85000 \mathrm{~K}$ for projectile ions with $Z_{p}>50$ at a few $\mathrm{MeV} / \mathrm{u}$. These electron temperatures may lead to materials modifications via the electronphonon coupling (thermal-spike model) or via the modified interatomic forces (lattice-relaxation model). A first analysis of the possible dependencies points to an influence of the electronic density of states (DOS) on the electron temperature. Large quasigaps at the Fermi level such as in $\mathrm{a}-\mathrm{C}, \mathrm{HOPG}$ and Be seem to yield the highest electron temperatures.

In summary, the Auger decay of multiple inner-shell vacancies yields snapshots of the track evolution for times between 1 and 20 fs. A significant ion-track potential seems to persist for some femtoseconds only in case of a few solids. High electron temperatures, however, seem to be a very general phenomenon. It is still an open question, whether materials modifications are triggered by the electronphonon coupling (thermal spike) or by the lattice relaxation (cold melting). So far, it has not been possible to distinguish between the two energyconversion mechanisms on a pure experimental basis. From the present work, however, it becomes clear that the pathways for materials modifications by fast heavy ions (Coulomb explosion versus thermal spike) are strongly dependent on the type of material.

\section{Acknowledgments}

This work was supported through the PROBRAL contract between DAAD and CAPES.

\section{References}

[1] R.L. Fleischer, P.B. Price, and R.M. Walker, 'Nuclear Tracks in Solids' (University of California Press, Berkely California, 1975).

[2] R. Spohr, 'Ion tracks and microtechnology' (F.Vieweg und Sohn Verlagsgesellschaft, Braunschweig 1990).

[3] S.Klaumünzer, Ming-dong Hou and G.Schumacher, Phys.Rev.Lett. 57, 850 (1986).

[4] Johnson RE and Brown WL., Nucl. Instr. and Meth. B198, 103-118 (1982)

[5] Akkermann A, Levinson J, Ilberg D and Lifshitz Y, Ionization of Solids by Heavy Particles ed. by R. Baragiola, NATO Advanced Study Institutes Series 306, Plenum Press, New York and London, 431-438 (1992)

[6] Leseur D and Dunlop A., Rad. Eff. and Def. Solids 126, 163-172 (1993)

[7] Watson CC and Tombrello TA., Radiation Effects 89, 263-283 (1985)

[8] P. Stampfli, K.H. Bennemann, Phys. Rev. B49, 7299 (1994); P. Stampfli, Nucl. Instr. Meth. B107, 138 (1996).

[9] F. Desauer, Z. Phys. 12, 38 (1923).

[10] I.M. Lifshitz, M.I. Kaganov and L.V. Tanatarov, J. Nucl. Energy A12, 69 (1960).

[11] Ritchie RH and Claussen C., Nucl. Instr. and Meth. B198, 133-138 (1982)

[12] Z.G. Wang, C. Dufour, E. Paumier and M. Toulemonde, J. Phys.: Condens. Matter 6, 6733 (1994).

[13] G. Szenes, Nucl. Instr. Meth. B116, 141 (1996).

[14] A.E. Volkov, V.A. Borodin, Nucl. Instr. Meth. B107, 172 (1996). 
[15] Birkhoff RD, in Handbuch der Physik, Vol. 34, ed by S.Flügge, Springer Verlag, Berlin, 53-138 (1958)

[16] M. Rösler and W. Brauer as well as D. Hasselkamp in 'Particle Induced Electron Emission', Springer tracts of modern physics, Vols. 123 and 123, Springer, Berlin (1991)

[17] Schiwietz G, in 'Ionization of Solids by Heavy Particles' ed by R. Baragiola, NATO Advanced Study Institutes Series 306, Plenum Press, New York and London, 197-214 (1992)

[18] Rothard H. (1995). Swift heavy ion induced electron emission from solids, Scanning Microscopy, Vol. 9, $1-42$

[19] H.D. Betz, Rev. of Modern Physics, 44, 465 (1972)

[20] H. Geissel, P. Armbruster, T. Kitahara, G. Kraft, H. Spieler, and K. Güttner, Nucl. Instr. Meth. 170, 217-219 (1980); H. Geissel, Y. Laichter, W. F. W. Schneider and P. Armbruster, Physics Letters A88, 26-28 (1982); H. Geissel, GSI report 82-12, ISSN:0171-4546 (Darmstadt, Germany, 1982); H. Geissel, H. Weick, C. Scheidenberger, R. Bimbot and D. Gardès Nucl. Instr. Meth. B195, 3-54 (2002).

[21] G. Schiwietz and P.L. Grande, Nucl. Instr. Meth. B175-177, 125-131 (2001); see also references therein

[22] H. Paul, Nucl. Instr. Meth. B, in print $(2003)$

[23] P.L. Grande and G.Schiwietz, Phys. Lett. A 163, 439 (1992); G. Schiwietz and P.L. Grande, Rad.Eff.and Def.in Solids 130/131, 137-156 (1994)

[24] P.M. Echenique, R.H. Ritchie and W.Brandt, Phys. Rev. B20, 2567 (1979); P.M. Echenique, R.M. Nieminem and R.H. Ritchie, Solid State Commun. 37, 779 (1981) P.M. Echenique, R.M. Nieminem, J.C.
Ashley and R.H. Ritchie, Phys. Rev. A33, 897 (1986)

[25] P.Bauer, F.Kastner, A.Arnau, A.Salin, P.D.Fainstein, V.H.Ponce, and P.M.Echenique, Phys. Rev. Lett. 69, 1137-1139 (1992); A. Arnau, P. Bauer, F. Kastner, A. Salin, V.H. Ponce, P.D. Fainstein, and P.M.Echenique, Phys. Rev. B49, 6470-6480 (1994)

[26] W. Fritsch and C.D. Lin, Phys. Rep. 202, 1-97 (1991)

[27] U.Wille and R.Hippler, Phys.Rep. 132, 129-260 (1986)

[28] H.Bethe, Ann.Phys. 5, 325 (1930)

[29] H. A. Bethe, R.W. Jackiw, ' Intermediate Quantum Mechanics' (2nd edition, W.A. Benjamin, inc, 1968).

[30] G. Schiwietz and P.L. Grande, Nucl.Instr. and Meth. B69, 1017 (1992); P.L. Grande and G.Schiwietz, Phys.Rev. A47 111922 (1993).

[31] G. Schiwietz, P.L. Grande, Radiation Effects and Defects in Solids 130/131, 137-156 (1994), and references therein.

[32] G. Schiwietz, P.L. Grande, C. Auth, H. Winter, and A. Salin, Phys.Rev.Lett. 72, 2159-62 (1994)

[33] P.L. Grande, G.Schiwietz, Phys. Rev. A58 , 3796-3801 (1998); G. Schiwietz, P.L. Grande, Nucl. Instr. Meth. B153, 1-9 (1999); G. de M. Azevedo, P.L. Grande, G. Schiwietz, Nucl. Instr. Meth. B164-165, 203 (2000).

[34] Version 1.3 of the CASP code for the UCA and PCA energy-loss theories including charge-state formulas with shell effects may be downloaded from "http://www.hmi.de/people/ schiwietz/casp.html".

[35] P. Sigmund, Nucl. Instr. Meth. B135, 1-15 (1998); A. Schinner, P. Sigmund, Nucl. Instr. Meth. B195, 64-90 (2002) 
[36] G. Maynard, M. Chabot, D. Gardes, Nucl. Instr. Meth. B164-165, 139146 (2000).

[37] A.F. Lifschitz, N.R. Arista , Phys. Rev. A (2003) in print.

[38] G. Xiao, Ph.D. thesis, ISBN 3928943-85-5, Freie Universität, Berlin, 1996; G. Schiwietz and G. Xiao (private communication); experimental equilibrium thicknesses for heavy ions at $6 \mathrm{MeV} / \mathrm{u}$ have recently been determined by Y. Sato et al., Nucl. Instr. Meth. B201, 571-580 (2003).

[39] J.P.Rozet, A.Chetioui, P.Bouisset, D.Vernhet, K.Wohrer, A.Touati, C.Stephan, and J.P.Grandin, Phys. Rev. Lett. 58, 337 (1987); J.P.Rozet, A.Chetioui, P. Piquemal, D. Vernhet, K. Wohrer, C.Stephan, and L.Tassan-Got, J.Phys. B 22, 33 (1989); J.P.Rozet, C.Stephan, D. Vernhet , Nucl. Instr. and Meth. B107, 67-70 (1996); M. Beuve, B. Gervais, E. Lamour, J. P. Rozet, D. Vernhet and L. J. Dubé, Phys. Lett. bf A274,37-46 (2000).

[40] N. Bohr, Phys. Rev. 58, 654 (1940); ibid 59, 270 (1941).

[41] V.S. Nikolaev and I.S. Dmitriev, Physics Letters 28A, 277 (1968).

[42] J.F. Ziegler, J.P. Biersack and U. Littmark, The Stopping and Range of Ions in Solids (Pergamon, New York, 1985).

[43] P. L. Grande and G. Schiwietz, Nucl. Instr. Meth. B195, 55-63 (2002)

[44] Extensive experimental energyloss tabulations are presented by H. Paul at 'http://www2.unilinz.ac.at/fak/TNF/atomphys/ STOPPING/welcome.htm'

[45] P.L. Grande, G. Schiwietz, J. Phys. B28, 425-433 (1995)

[46] D.Schneider, G.Schiwietz, and D.DeWitt, Phys. Rev. A47, 39453950 (1992)
[47] G. Schiwietz, E. Luderer, K. Czerski, M. Roth, F. Staufenbiel, P.L. Grande, Nucl. Instr. Meth. B193, 705-712 (2002); the assignment of a $2 \mathrm{pVV}$ surface-plasmon peak is false as discussed in ref. [54]; Auger shift and intensity results are superseded by the present work

[48] C.J.Powell and A.Jablonski, "NIST electron inelastic-mean-free-path database", reference database 71 , version 1.1, National Institute of Standards and Technology, USA

[49] G. Schiwietz, G. Xiao, Nucl. Instr. Meth. B107, 113 (1996).

[50] G. Schiwietz, E. Luderer, G. Xiao and P.L. Grande, Nucl. Instr. Meth. B175-177, 1-11 (2001).

[51] G. Schiwietz, D. Schneider, J.P. Biersack, N. Stolterfoht, D. Fink, A. Mattis, B. Skogvall, H. Altevogt, V. Montemayor, U. Stettner, Phys. Rev. Lett. 61, 2677 (1988)

[52] G. Xiao, G. Schiwietz, P.L. Grande, A. Schmoldt, N. Stolterfoht, M. Grether, R. Köhrbrück, A. Spieler and U. Stettner, Phys. Rev. Lett. 79, 1821 (1997).

[53] M. Krause, J. Phys. Chem. Ref. Data 8, 307 (1979); M.Krause and J.H.Oliver, J.Phys.Chem.Ref.Data 8, 329 (1979)

[54] G. Schiwietz, M. Roth, K. Czerski, F. Staufenbiel, M. Rösler, and P.L. Grande, Nucl. Instr. Meth. B209, 26-31 (2003)

[55] W. Schmidt, P. Müller, V. Brückner, F. Löffler, G. Saemann-Ischenko, W. Schubert, Phys. Rev. A24, 2420 (1981).

[56] A. Koyama, H. Ishikawa, K. Maeda, Y. Sasa, O. Benka, M. Uda, Nucl. Instr. Meth. B48, 608 (1990).

[57] M. Caron, H. Rothard, M. Beuve, B. Gervais, Physica Scipta T80, 331 (1999); - ibid T92, 281 (2001). 
[58] J.C. Fuggle and S.F. Alvarado, Phys. Rev. A22, 1615 (1980).

[59] 7 measured values for the Si-2p lifetime deviate from each other and from ref. [53] by up to a factor of 6 . The value of $15 \mathrm{fs}$ results from an average of the 5 most reliable results.

[60] M.Rösler and W.Brauer, 'Particle Induced Electron Emission I', Springer tracts of modern physics, Vol. 122, (Springer, Berlin,1991).

[61] G. Schiwietz, P.L. Grande, B. Skogvall, J.P. Biersack, R. Köhrbrück, K. Sommer, A. Schmoldt, P. Goppelt, I. Kádár, S. Ricz, and U. Stettner, Phys. Rev. Lett. 69, 628 (1992).

[62] K. Wien, Ch. Koch, and Nguyen van Tan, Nucl. Instr. Meth. B100, 322 (1995).

[63] G. Galli, R.M. Martin, R. Car, M. Parrinello, Phys. Rev. B42, 7470 (1990); J. Schäfer, J. Ristein, R. Graupner, L. Ley, U. Stephan, Th. Frauenheim, V.S. Veerasamy, G.A.J. Amaratunga, M. Weiler, H. Erhardt, Phys. Rev. B53, 7762 (1996).

[64] G. Schiwietz, G. Xiao, P.L. Grande, E. Luderer, R. Pazirandeh, U. Stettner, Nucl. Instr. Meth. B146, 131136 (1998); - ibid, Europhys. Lett. 47, 384-390 (1999), G. Schiwietz, G. Xiao, E. Luderer, and P.L. Grande, Nucl. Instr. Meth. B164, 353-364 (2000).

[65] S. Tougaard and P. Sigmund, Phys. Rev. B25, 4452-66 (1982).

[66] S.K. Bose et al., Phys. Rev. B37, 6262 (1988);

[67] S. Eisebitt et al., J. Electron Spectr. Rel. Phenomena 93, (1998) 245

[68] H. Haas, calulations for the partial density of states of $\mathrm{Si}$ using the FLAPW electronic structure code WIEN, private communication (2003)
[69] G. de M. Azevedo, P. L. Grande, M. Behar, J.F. Dias, and G. Schiwietz, Phys.Rev.Lett. 86, 1482-1485 (2001); G. de M. Azevedo, M. Behar, J.F Dias , P. L. Grande, D. L. da Silva, and G. Schiwietz, Phys.Rev. B65, 075203 (2002); L. L. Araujo , P.L. Grande, M. Behar, J.F. Dias , J.H.R. dos Santos, G. Schiwietz, Nucl. Instr. Meth. B193, 172-177 (2002)

[70] H.-D. Betz, D. Röschenthaler and J. Rothermel, Phys. Rev. Lett. 50, 34 (1983)

[71] K. Dybdal, J. Sørensen, P. Hvelplund and H. Knudsen, Nucl. Instr. Meth. bf B13, 581-586 (1986)

[72] G. Schiwietz, D. Schneider, J. Tanis, Phys. Rev. Lett. 59, 1561-1564 (1987); G. Schiwietz, Radiation Effects and Defects in Solids 112, 195200 (1990).

[73] H. J. Hay and P. B. Treacy, Nucl. Instr. Meth. bf B48, 107-110 (1990)

[74] K. Czerski et al., in preparation (2003); K. Czerski, F. Staufenbiel, M. Roth, G. Schiwietz, and P.L. Grande, pp. 12-13 ISL annual report 2002, (Berlin, ISSN 1610-0638); see also the contribution on electronic sputtering of $\mathrm{BeO}$ in this topical issue

[75] F. Staufenbiel, Ph.D. thesis, in preparation (2003); F. Staufenbiel, K. Czerski, M. Roth, G. Schiwietz, pp. 14-15, ISL annual report 2002, (Berlin, ISSN 1610-0638) 